\title{
Esito del prolattinoma dopo la gravidanza e l'allattamento: uno studio su 73 pazienti
}

\author{
Salvatore Cannavò
}

Pubblicato online: 17 luglio 2014

(C) Springer International Publishing AG 2014

\section{Commento a:}

Outcome of prolactinoma after pregnancy and lactation: a study on 73 patients. M. Domingue, F. Devuyst, O. Alexopoulou, B. Corvilain, D. Maiter.

\section{Clin Endocrinol (2014) 80:642-648}

I prolattinomi sono i tumori ipofisari più frequentemente diagnosticati, con una prevalenza di 30-50 casi per 100.000 abitanti. L' $80-90 \%$ dei prolattinomi sono diagnosticati in donne, spesso giovani. I dopamino-agonisti rappresentano la terapia di prima linea, capace di ridurre sia la secrezione di PRL che il volume tumorale, con effetti benefici sulla fertilità. Pertanto, una gravidanza non è un evento raro nelle donne con prolattinoma. Per questo motivo, la gestione del prolattinoma durante la gravidanza, così come quella della gravidanza nelle donne con prolattinoma, sono stati argomenti ampiamente discussi negli anni recenti, con studi focalizzati sui rischi cui va incontro il feto o sul rischio di incremento volumetrico del tumore ipofisario. Meno conosciuta è invece la storia naturale del prolattinoma dopo la gravidanza e dopo l'allattamento. In passato, alcuni studi hanno riportato la normalizzazione dei livelli di PRL dopo il parto nel 10-68\% delle donne con iperprolattinemia funzionale o tumorale, ma pochissimi dati sono disponibili sugli effetti dell'allattamento, sebbene esso venga considerato sufficientemente sicuro.

Lo studio di Domingue et al. riporta un' analisi retrospettiva condotta durante 20 anni (dal 1992 al 2012) in due centri universitari del Belgio su 73 donne con micro- o macro-

S. Cannavò $(\varangle)$

Dipartimento di Medicina Clinica e Sperimentale, Università di

Messina, Messina, Italia

e-mail: cannavos@unime.it prolattinoma, che sono andate incontro a 104 gravidanze. Settanta donne praticavano dopamino-agonisti al momento del concepimento (cabergolina nel $96 \%$ dei casi), che sono stati sospesi durante il primo trimestre di gravidanza. Le donne che erano in remissione dopo una precedente gravidanza o che hanno dovuto riprendere il trattamento con dopamino-agonisti dopo la gravidanza sono state escluse dallo studio.

Delle 104 gravidanze, 13 sono state considerate a rischio, 7 sono state gemellari, 3 si sono interrotte dopo il primo trimestre e 10 sono esitate in parto prematuro. I livelli di PRL si sono mantenuti nella norma durante il follow-up (mediana 22 mesi) nel $41 \%$ delle donne che avevano partorito e/o allattato. Le dimensioni dell'adenoma ipofisario dopo la gravidanza e l'allattamento si sono ridotte nel 63\% dei casi. Esse erano minori nel gruppo delle donne che sono andate incontro a una prolungata remissione di malattia, con una più elevata, anche se non significativamente, prevalenza di remissioni nel gruppo con microprolattinoma. Nel $23 \%$ delle pazienti la RMN non ha più individuato il tumore ipofisario dopo il parto e nel $39 \%$ le sue dimensioni erano comunque ridotte. La remissione dell'iperprolattinemia non era influenzata dal numero di gravidanze né dalla durata dell'allattamento.

In conclusione, questo studio ha dimostrato che oltre il $40 \%$ delle donne che vanno incontro a gravidanza durante l'assunzione di dopamino-agonisti per diagnosi di prolattinoma dimostrano normali livelli di PRL a distanza di quasi 2 anni dopo il parto e/o l'allattamento. La probabilità di mantenere una stabile normalizzazione dei livelli ormonali è associata a un diametro minore dell'adenoma al momento della diagnosi e alla scomparsa dell'adenoma alla RMN dopo la gravidanza. 